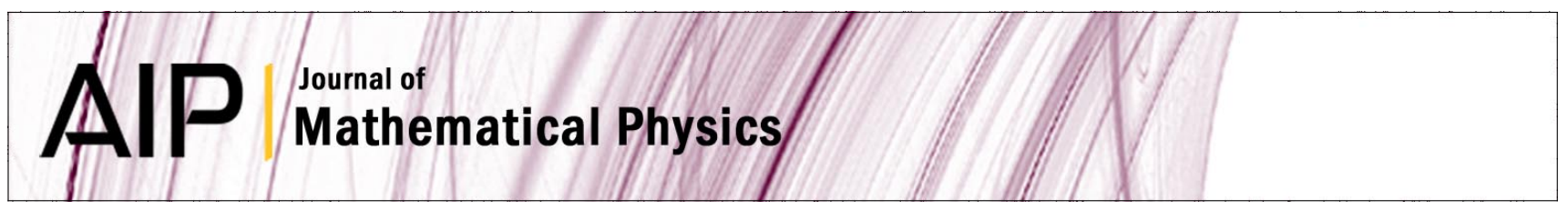

\title{
Boundary value problems for integrable equations compatible with the symmetry algebra
}

Burak Gürel, Metin Gürses, and Ismagil Habibullin

Citation: J. Math. Phys. 36, 6809 (1995); doi: 10.1063/1.531189

View online: http://dx.doi.org/10.1063/1.531189

View Table of Contents: http://jmp.aip.org/resource/1/JMAPAQ/v36/i12

Published by the American Institute of Physics.

\section{Additional information on J. Math. Phys.}

Journal Homepage: http://jmp.aip.org/

Journal Information: http://jmp.aip.org/about/about_the_journal

Top downloads: http://jmp.aip.org/features/most_downloaded

Information for Authors: http://jmp.aip.org/authors

\section{ADVERTISEMENT}

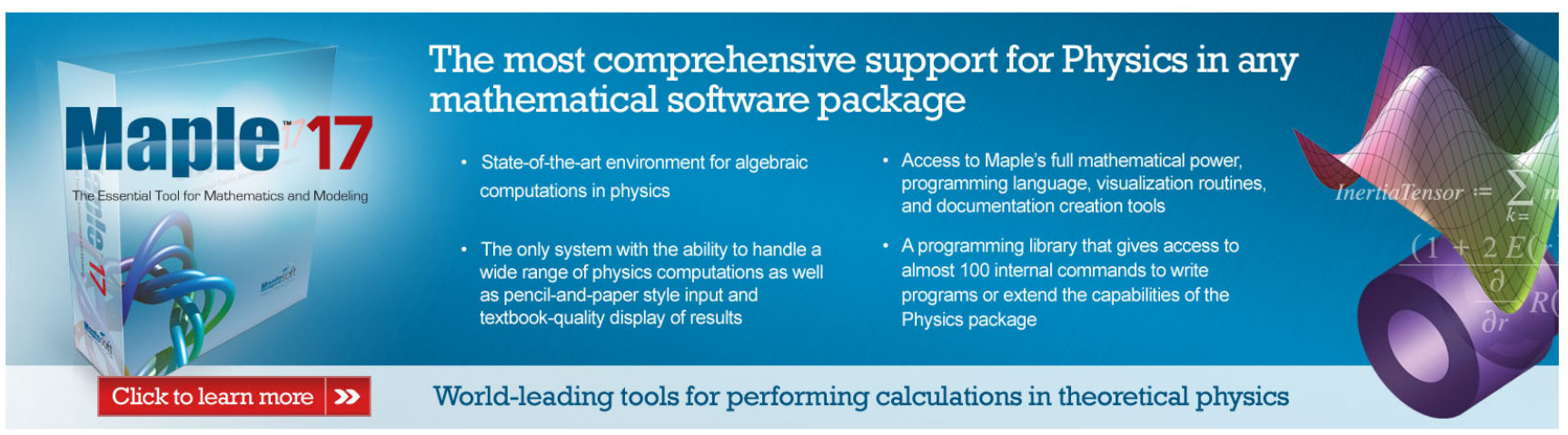




\title{
Boundary value problems for integrable equations compatible with the symmetry algebra
}

\author{
Burak Gürel and Metin Gürses \\ Department of Mathematics, Faculty of Science, Bilkent University, 06533 Ankara, Turkey \\ Ismagil Habibullin \\ Mathematical Institute, Ufa Scientific Center, Russian Academy of Sciences, \\ Chernishevski str. 112, Ufa, 450000, Russia
}

(Received 2 December 1994; accepted for publication 17 July 1995)

Boundary value problems for integrable nonlinear partial differential equations are considered from the symmetry point of view. Families of boundary conditions compatible with the Harry-Dym, $\mathrm{KdV}$, and $\mathrm{mKdV}$ equations and the Volterra chain are discussed. We also discuss the uniqueness of some of these boundary conditions. (C) 1995 American Institute of Physics.

\section{INTRODUCTION}

In our previous paper $^{1}$ we have briefly discussed a method to construct boundary value problems of the form

$$
\begin{gathered}
u_{t}=f\left(u, u_{1}, u_{2}, \ldots, u_{n}\right), \\
\left.p\left(u, u_{1}, u_{2}, \ldots, u_{k}\right)\right|_{x=0}=0,
\end{gathered}
$$

completely compatible with the integrability property of Eq. (1). Here $u=u(x, t), u_{i}=\partial^{i} u / \partial x^{i}$ and $f$ is a scalar (or vector) field. The aim of the present paper is to expound in detail our scheme and also extend it to the integrable differential-difference equations.

Let the equation

$$
u_{\tau}=g\left(u, u_{1}, \ldots, u_{m}\right),
$$

for a fixed value of $m$, be a symmetry of Eq. (1). Let us introduce some new set of dynamical variables, consisting of the variable $v=\left(u, u_{1}, u_{2}, \ldots, u_{n-1}\right)$, and its $t$-derivatives $v_{t}, v_{t t}, \ldots$. One can express the higher $x$-derivatives of $u$, i.e., $u_{i}$ for $i \geqslant n$ and their $t$-derivatives, by using Eq. (1), in terms of the dynamical variable $v$ and their $t$-derivatives. Here $n$ is the order of Eq. (1). In these terms the symmetry (3) may be written as

$$
v_{\tau}=G\left(v, v_{t}, v_{t}, \ldots, v_{t t \cdots t}\right) .
$$

We call the boundary value problem, Eqs. (1) and (2), as compatible with symmetry (3) if the constraint $p(v)=0$ [or constraints $p^{a}(v)=0$, where $a=1,2, \ldots, N$ and $N$ is the number of constraints] is consistent with the $\tau$-evolution,

$$
\frac{\partial p}{\partial \tau}=0 \quad(\bmod p=0)
$$

Equation (5), by virtue of the equations in (4), must be automatically satisfied. In fact, (5) means that the constraint $p=0$ defines an invariant surface in the manifold with local coordinates $v$. This definition of consistency of the boundary value problem with symmetry is closer to the one introduced in Ref. 2, but not identical. 
We call the boundary condition (2) compatible with the equation if it is compatible at least with one of its higher-order symmetries.

Our main observation is that if the boundary condition is compatible with one higher symmetry, then it is compatible with an infinite number of symmetries that form a set $S$ with an infinite elements. Here $S$ may or may not contain the whole symmetries of (1). For instance, $S$ contains the even-ordered time-independent symmetries for the Burgers' equation.

We note that all the known boundary conditions of the form (2) consistent with the inverse scattering method are indeed compatible with the infinite series of generalized symmetries. On the other hand, stationary solutions of the symmetries compatible with (2) allow one to construct an infinite-dimensional set of "exact" (finite gap) solutions of the corresponding boundary value problem (1) and (2). However, in this work we do not discuss analytical aspects of this problem. We note also that, in this paper we shall deal with boundary conditions of the form given in (2). An effective investigation of boundary conditions involving an explicit $t$-dependence is essentially more complicated. Such a problem has been studied, for instance, in Ref. 3.

The paper is organized as follows. In Sec. II we present some propositions related to the boundary conditions compatible with the infinite number of higher symmetries and prove them. As an illustrative example we find all possible boundary conditions discussed in Sec. II of the Burgers' equation in Sec. III. In Sec. IV we consider the nonlinear Schrödinger, Harry-Dym, Korteweg de Vries, and modified KdV equations. Using the symmetry approach we find a boundary condition compatible with the symmetry algebra of the Harry-Dym equation,

$$
u_{t}=u^{3} u_{x x x}, \quad u_{x}=c u, \quad x=0, \quad u_{x x}=c^{2} u / 2, \quad x=0,
$$

where $c$ is an arbitrary real constant. Actually one has here two constraints. Although we are taking the boundary conditions at $x=0$, one can shift this point to an arbitrary point $x=x_{0}$ without losing any generality. We conjecture that the boundary value problem given in (6) is compatible with the Hamiltonian integrability and solvable by the inverse scattering technique. In addition, we conjecture that (using the idea in Ref. 4) one can prove that on the finite interval $x_{1} \leqslant x \leqslant x_{2}$ the Harry-Dym equation with the boundary conditions $u_{x}=c_{0} u, u_{x x}=c_{0}^{2} u / 2$ for $x=x_{1}$ and $u_{x}=c_{1} u$, $u_{x x}=c_{1}^{2} u / 2$ for $x=x_{2}$ is a completely integrable Hamiltonian system.

Section $\mathrm{V}$ is devoted to the differential-difference equations. In the last section we propose a further generalization of the compatibility and discuss some open questions.

\section{BOUNDARY CONDITIONS COMPATIBLE WITH SYMMETRIES}

In the sequel we suppose that Eq. (1) admits a recursion operator of the form (see Refs. 5-7)

$$
R=\sum_{i=0}^{i_{1}} \alpha_{i} D^{i}+\sum_{i=0}^{k_{1}} \alpha_{-1, i} D^{-1} \alpha_{-2, i}, \quad i_{1} \geqslant 0, \quad k_{1} \geqslant 0,
$$

where $\alpha_{i}, \alpha_{-1, i}, \alpha_{-2, i}$ are functions of the dynamical variables, $D$ is the total derivative with respect to $x$, and $D^{-1}$ is defined through the relation

$$
\left(D^{-1} \varphi\right)(x)=\int_{-\infty}^{x} \varphi(\xi) d \xi .
$$

Recursion operators when applied to a symmetry produce new symmetries. Passing to the new dynamical variables $v, v_{t}, v_{t t}, \ldots$, one can obtain, from (7), the recursion operator $\mathbf{R}$ of the system of equations (4) (we do not prove that every recursion operator may be rewritten in the matrix form, but we will give below the matrix forms of the recursion operators for the Burgers', KdV, $\mathrm{mKdV}$, and Harry-Dym equations), 


$$
\mathbf{R}=\sum_{i=0}^{M} a_{i}\left(\partial_{t}\right)^{i}+\sum_{i=0}^{K} a_{-1, i}\left(\partial_{t}^{-1}\right) a_{-2, i}, \quad M>0, \quad K \geqslant 0,
$$

where the coefficient matrices $a_{i}, a_{-1, i}, a_{-2, i}$ depend on $v$ and on a finite number of its $t$-derivatives, and $\partial_{t}$ is the operator of the total derivative with respect to $t$. If (1) is a scalar equation, $R$ is a scalar operator, then $\mathbf{R}$ is an $n \times n$ matrix valued operator. Our further considerations are based on the following proposition, which really affirms that if an equation admits an invariant surface, then an infinite number of its higher symmetries admits also the same invariant surface.

Proposition 2.1: Let Eq. (4) be of the form $v_{\tau}=T(\mathbf{R}) v_{t}$ where $\mathbf{R}$ is the recursion operator (8) and $T$ is a polynomial function with scalar constant coefficients. If this equation is consistent with the constraint $p(v)=0$, where rank of $p$ equals $n-1$ (here $n$ is the dimension of the vector $v$ ), then every equation of the form $v_{\tau}=L(T(\mathbf{R})) v_{t}$, where $L$ is arbitrarily chosen polynomial with scalar constant coefficients, is also compatible with this constraint.

Proof: Introduce new variables $w=\left(w^{1}, w^{2}, \ldots, w^{n}\right)$ in the following way: $w^{1}=p^{1}$, $w^{2}=p^{2}, \ldots, w^{n-1}=p^{n-1}$, and $w^{n}=p^{n}$ is a function of $v$; here $p^{i}$ are the components of the vector $p$ for $i \leqslant n-1$. Then one obtains the equation $w_{\tau}=P w_{t}$ from (5), where $P=A T(\mathbf{R}) A^{-1}$ and $A=\partial w / \partial v$ is the Jacobi matrix of the mapping $v \rightarrow w$. Notice that under this change of variables the constraint $p(v)=0$ turns into the equation $w^{i}=0$ for $i=1,2, \ldots, n-1$. Imposing this constraint reduces the equation $w_{\tau}=P w_{t}$ to the form

$$
\left(\begin{array}{c}
0 \\
\cdots \\
0 \\
w_{\tau}^{n}
\end{array}\right)=\left(\begin{array}{ccc}
P_{11} & \cdots & P_{1 n} \\
\cdots & \cdots & \cdots \\
P_{n-1,1} & \cdots & P_{n-1, n} \\
P_{n, 1} & \cdots & P_{n, n}
\end{array}\right)\left(\begin{array}{c}
0 \\
\cdots \\
0 \\
w_{t}^{n}
\end{array}\right) .
$$

Let us show that elements of the last column of the matrix $P$ are equal to zero except maybe $P_{n, n}$ : $P_{i, n}=0$ for $1 \leqslant i \leqslant n-1$. Really, by letting $P_{j, n} \neq 0$ for some $j \leqslant n-1$ the equation $P_{j, n} w_{t}^{n}=0$ gives a connection between variables $w^{n}, w_{t}^{n}, \ldots$, which are supposed to be independent. The set of such operator valued matrices with

$$
\sum_{j=1}^{n-1} P_{i j}(0)=0, \quad \forall i=1,2, \ldots, n-1
$$

constitutes a subalgebra $M^{*}$ in the algebra of all square matrices; hence one can easily conclude that the operator $L(P)\left(\bmod w^{i}=0, i \leqslant n-1\right)$ is in $M^{*}$, so the equation $w_{\tau}=L(P) w_{t}$ is consistent with the constraint $w^{i}=0, i \leqslant n-1$. It completes the proof of Proposition 2.1.

Proposition 2.2: Suppose that $p(v)=0$ is set of constraints of rank $n-1$ and that there exists a positive integer $n_{0}$ such that the coefficient matrix $b_{M}$ in the expression $\mathbf{R}^{n_{0}}=b_{M}\left(\partial_{t}\right)^{M}$ $+b_{M-1}\left(\partial_{t}\right)^{M-1}+\cdots$ is proportional to the identity matrix. Then $p(v)=0$ is compatible with the symmetry $v_{\tau}=\mathbf{R}^{n_{0}} v_{t}$ if and only if it is compatible with the symmetry $v_{\tau}=H\left(\mathbf{R}^{n_{0}}\right) v_{t}$, where $H$ is a polynomial with scalar constant coefficients.

Proof: Assume $p(v)=0$ is compatible with $v_{\tau}=H\left(\mathbf{R}^{n_{0}}\right) v_{t}$. In terms of the variable $w$ we have introduced proving the previous proposition, the equation $v_{\tau}=H\left(\mathbf{R}^{n_{0}}\right) v_{t}$ takes the form $w_{\tau}$ $=H\left(R_{1}^{n_{0}}\right) w_{t}$. Owing to the fact that the point transformation preserves the commutativity property of flows, the operator $R_{1}=A \mathbf{R} A^{-1}$ is the recursion operator in the new variables. Again, just in the previous proposition one has that the operator $H\left(R_{1}^{n_{0}}\right)$ under the substitution $p=0$ (or really, $\left.w^{i}=0, i \leqslant n-1\right)$ belongs to the subalgebra $M^{*}$. Our aim now is to prove that the operator $Q$ $=R_{1}^{n_{0}}\left(\bmod w^{i}=0, i \leqslant n-1\right)$ is in $M^{*}$. Setting $H(Q)=\alpha_{n} Q^{n}+\alpha_{n-1} Q^{n-1}+\cdots+\alpha_{0}$ and representing $Q$ as formal series $\sum_{k=-\infty}^{M} c_{k} \partial_{t}^{k}$ using the famous Campbell-Hausdorff formula, one obtains that 


$$
H(Q)=\alpha_{n}\left[c_{M}^{n}\left(\partial_{t}\right)^{n M}+n c_{M}^{n-1} c_{M-1}\left(\partial_{t}\right)^{n M-1}+\cdots\right]+\cdots+\alpha_{0} .
$$

One has that $H(Q)$ belongs to the subalgebra $M^{*}$. By looking at the coefficients of different power of the operator $\partial_{t}$, one can show that the matrices $c_{i}, i=M-1, M-2, \ldots$, satisfy the equations

$$
c_{M}^{n-1} c_{i}+S_{i} \in M^{*},
$$

where $S_{i}$ are polynomials with scalar coefficients on variables $c_{i+1}, c_{i+2}, \ldots, c_{M}$ and their derivatives. So, because of assumptions $c_{M}=b_{M} \in M_{0}$, where $M_{0}$ is the set of all matrices proportional to the identity matrix, and $\operatorname{det} b_{M} \neq 0$, it is easy to prove by induction that $c_{i} \in M_{0}$ for all $i \leqslant M$.

Assume $p(v)=0$ is compatible with $v_{\tau}=\mathbf{R}^{n_{0}} v_{t}$. Now let the polynomial $T$ in Proposition 2.1 be $T(z)=z^{n_{0}}$. So the proof is completed.

\section{BOUNDARY CONDITIONS OF THE BURGERS' EQUATION}

For the application of the propositions given in the previous section, in particular Proposition 2.2 , we study the Burgers' equation in detail as an example. It has some special importance. We can find all possible boundary conditions compatible with the even-ordered generalized symmetries. The Burgers' equation and its recursion operator are, respectively, given by, e.g., in Ref. 8,

$$
\begin{gathered}
u_{t}=u_{x x}+2 u u_{x}, \\
R=D+u+u_{x} D^{-1} .
\end{gathered}
$$

The simplest symmetry of this equation is $u_{\tau}=u_{x}$. In terms of the new dynamical variables, this symmetry equation takes the form

$$
u_{\tau}=u_{1}, \quad u_{1, \tau}=u_{t}-2 u u_{1} .
$$

This equation does not admit any invariant surface of the form $p\left(u, u_{1}\right)=0$. Really, differentiating this constraint with respect to $\tau$, one obtains

$$
\frac{\partial p}{\partial u} u_{1}+\frac{\partial p}{\partial u_{1}}\left(u_{t}-2 u u_{1}\right)=0 .
$$

Because of independence of the variables $u_{t}$ and $u_{1}$, we have

$$
\frac{\partial p}{\partial u_{1}}=\frac{\partial p}{\partial u}=0,
$$

which leads to a trivial solution $p=$ const. As a conclusion we do not have any invariant surface (curve) in the $\left(u, u_{1}\right)$ plane. Similarly, the third-order symmetry $u_{\tau}=u_{3}+3 u u_{2}+3 u_{1}^{2}+3 u^{2} u_{1}$ rewritten in the new variables $\left(u, u_{1}\right)$ gives the following system of two equations:

$$
\begin{gathered}
u_{\tau}=u_{1, t}+u u_{t}+\left(u^{2}+u_{1}\right) u_{1}, \\
u_{1, \tau}=u_{t t}-u u_{1, t}+\left(u^{2}+u_{1}\right) u_{t}-2 u u_{1}\left(u^{2}+u_{1}\right) .
\end{gathered}
$$

This system also does not admit any invariant surface of the form $p\left(u, u_{1}\right)=0$. It may be easily proved that the same is true for every symmetry of the odd order, i.e., $u_{r}=u_{2 m+1}+h\left(u_{2 m}, \ldots, u\right)$. Because the correspondent system of equations has different orders in the highest $t$-derivatives,

$$
u_{\tau}=\partial_{t}^{m} u_{1}+\cdots, \quad u_{1, \tau}=\partial_{t}^{m+1} u+\cdots .
$$


Unlike the symmetries of odd order, for the symmetries of even order the correspondent system of equations has the same orders in the highest $t$-derivatives. This fact leads us to show that the symmetries of even order admit an invariant surface $p\left(u, u_{1}\right)=0$, depending upon two arbitrary parameters.

Proposition 3.1: If the boundary condition $\left.p\left(u, u_{1}\right)\right|_{x=0}=0$ is compatible with a higher symmetry of the Burgers' equation, then it is of the form (see Ref. 2) $c\left(u_{1}+u^{2}\right)+c_{1} u+c_{2}=0$, and is compatible with every symmetry of the form $u_{\tau}=P\left(\mathbf{R}^{2}\right) u_{t}$, where $P$ denotes polynomials with scalar constant coefficients.

Proof: The Frechet derivative of (9) gives the symmetry equation of the Burgers' equation,

$$
\partial_{t} \sigma=\left(D^{2}+2 u D+2 w\right) \sigma
$$

where $w$ stands for $u_{1}$. Our aim is to express the recursion operator in terms of $\partial_{t}, \partial_{t}^{-1}$. To this end we rewrite (16) in the form $\partial_{t} \sigma=D(D+2 u) \sigma$, which is equivalent to

$$
D^{-1} \sigma=\partial_{t}^{-1}(D+2 u) \sigma
$$

Since the operators are acting on the symmetries, we may take

$$
D^{-1}=\partial_{t}^{-1}(D+2 u)
$$

in the recursion operator (10). Consequently, the recursion formula $u_{\tau_{i+1}}=R u_{\tau_{i}}$ becomes

$$
u_{\tau_{i+1}}=\left(u+2 w \partial_{t}^{-1} u\right) u_{\tau_{i}}+\left(1+w \partial_{t}^{-1}\right) w_{\tau_{i}} .
$$

Differentiating it with respect to $x$ and replacing $w_{x}=u_{2}=u_{t}-2 u w$, one obtains

$$
w_{\tau_{i+1}}=\left[\partial_{t}+2\left(u_{t}-2 u w\right) \partial_{t}^{-1} u\right] u_{\tau_{i}}+\left[-u+\left(u_{t}-2 u w\right) \partial_{t}^{-1}\right] w_{\tau_{i}},
$$

for $i=1,2, \ldots$. Thus the matrix form of the recursion operator $\mathbf{R}$ is given by

$$
\mathbf{R}=\left(\begin{array}{cc}
u+2 w \partial_{t}^{-1} u & 1+w \partial_{t}^{-1} \\
\partial_{t}+2\left(u_{t}-2 u w\right) \partial_{t}^{-1} u & -u+\left(u_{t}-2 u w\right) \partial_{t}^{-1}
\end{array}\right) .
$$

It is well known that every higher-order local polynomial symmetry may be represented as a polynomial operator $P_{0}(\mathbf{R})$ applied to the simplest classical symmetry $u_{\tau}=u_{x}$. It is more convenient to use the following equivalent representation:

$$
\left(\begin{array}{l}
u \\
w
\end{array}\right)_{\tau}=P\left(\mathbf{R}^{2}\right)\left(\begin{array}{l}
u \\
w
\end{array}\right)_{t}+P_{1}\left(\mathbf{R}^{2}\right)\left(\begin{array}{c}
w \\
u_{t}-2 u w
\end{array}\right),
$$

where $P$ and $P_{1}$ are polynomials with scalar constant coefficients and $P_{0}$ mentioned above may be taken as

$$
P_{0}(\mathbf{R})=P\left(\mathbf{R}^{2}\right) \mathbf{R}+P_{1}\left(\mathbf{R}^{2}\right)
$$

Note that one could not apply immediately Proposition 2.2 to this because the coefficient of $\partial_{t}$ in the representation (20) is not diagonal. On the other hand, the operator $\mathbf{R}^{2}$ has a scalar leading part. First, we will prove that if the symmetry $(21)$ admits an invariant surface then $P_{1}$ in this equation vanishes. Let us take the invariant surface as $u=q(w)$. Suppose that the function $q(w)$ is differentiable at some point $w=w_{0}$. Linearizing $q$ around the point $w_{0}$ (or as $w \rightarrow w_{0}$ ), we obtain

$$
u-q\left(w_{0}\right)=q^{\prime}\left(w_{0}\right)\left(w-w_{0}\right)+o\left(w-w_{0}\right) .
$$


It follows from (20) that in this case $\mathbf{R}^{2}$ reduces to a scalar operator: $\mathbf{R}^{2} \rightarrow\left[\partial_{t}-w_{0}+q^{2}\left(w_{0}\right)\right] I$ as $w \rightarrow w_{0}$, where $I$ is the unit matrix. Thus, in the linear approximation Eq. (21) takes the form

$$
\left(\begin{array}{c}
u \\
w
\end{array}\right)_{\tau}=P\left[\partial_{t}-w_{0}+q^{2}\left(w_{0}\right)\right]\left(\begin{array}{c}
u \\
w
\end{array}\right)_{t}+P_{1}\left[\partial_{t}-w_{0}+q^{2}\left(w_{0}\right)\right]\left(\begin{array}{l}
w \\
u_{t}
\end{array}\right)
$$

where now $P\left[\partial_{t}-w_{0}+q^{2}\left(w_{0}\right)\right]$ and $P_{1}\left[\partial_{t}-w_{0}+q^{2}\left(w_{0}\right)\right]$ are scalar operators. It is clear that the linearized equation is consistent with the linearized boundary condition $u-q\left(w_{0}\right)=q^{\prime}\left(w_{0}\right)\left(w-w_{0}\right)$, provided $P_{1}=0$. Supposing that Eq. (21) is compatible with the constraint $w=c$ where $c$ is a constant and then linearizing about the point $(u=0, w=c)$, one can easily obtain that $P_{1}$ vanishes in this case also.

It is evident now that in Proposition 2.2 one should put $n_{0}=2$, because $\mathbf{R}^{2}=I \partial_{t}+\cdots$. With this choice the constraint $p(u, w)$ describes an invariant surface for the following system:

$$
\left(\begin{array}{l}
u \\
w
\end{array}\right)_{\tau}=\mathbf{R}^{2}\left(\begin{array}{c}
u \\
w
\end{array}\right)_{t}
$$

which is exactly the coupled Burgers' type integrable system (see Ref. 5),

$$
u_{\tau}=u_{t t}+2\left(w+u^{2}\right) u_{t}, \quad w_{\tau}=w_{t t}+2 u_{t}^{2}+2\left(w+u^{2}\right) w_{t} .
$$

It is straightforward to show that the above system (24) is compatible with the constraint $p(u, w)=0$ only if $p=w+u^{2}+c_{1} u+c_{2}$ or $u=$ const.

The above uniqueness proof of the boundary condition $p=w+u^{2}+c_{1} u+c_{2}$ can be more easily shown if we use a new property of the Burgers' hierarchy. We have the following proposition.

Proposition 3.2: The function $u\left(t, x, \tau_{n}\right)$, for $n \geqslant-1$, satisfy infinitely many Burgers'-like equations,

$$
u_{, \tau_{i}, \tau_{i}}-u_{, \tau_{2 i+2}}=-2 u_{, \tau_{i}} D^{-1} u_{, \tau_{i}},
$$

for all $i=-1,0,1,2, \ldots$.

Burgers' equation corresponds to $i=-1\left(\tau_{-1}=x\right.$ and $\left.\tau_{0}=t\right)$. All $u_{\tau_{i}}$ for $i>-1$ correspond to higher symmetries. Using this relation it is straightforward to determine the even numbered symmetries of the Burgers equation from (25). It is very interesting that $u$ satisfies the Burgers'-like equations with respect to the variables $\left(\tau_{i}, \tau_{2 i+2}\right)$ for all $i=-1,0,1,2, \ldots$.

The proof of this proposition depends crucially on definition of the higher symmetries of the Burgers' equation. They are defined through the equation

$$
u_{\tau_{n}}=R^{n+1} u_{x},
$$

where $R$ is the recursion operator given in Eq. (10) and $n \geqslant-1$. Equation (26) can also be written as $u_{\tau_{n}}=R u_{\tau_{n-1}}$. Differentiating this equation once by $\tau_{n}$ and using (26), one arrives at (25).

If we let the most general boundary condition of the form $p=f\left(u, u_{x}\right)=0$ at $x=x_{0}$ and take $\tau_{i}$ and $\tau_{2 i+2}$ derivatives for $i \geqslant 0$ of the function $p$ and use Eq. (25), we obtain

$$
f_{u_{x}}^{2} f_{, u, u}+f_{u}^{2} f_{, u_{x}, u_{x}}-2 f_{, u_{x}}^{3}-2 f_{, u} f_{, u_{x}} f_{u, u_{x}}=0 .
$$

Letting $u=x_{1}$ and $u_{x}+u^{2}+c_{1} u+c_{2}=x_{2}$, then Eq. (27) becomes

$$
f_{, x_{2}}^{2} f_{, x_{1}, x_{1}}+f_{, x_{1}}^{2} f_{, x_{2}, x_{2}}-2 f_{, x_{1}} f_{, x_{2}} f_{, x_{1}, x_{2}}=0 .
$$


Assuming $f_{x_{2}} \neq 0$ and letting $q=f_{, x_{1}} / f_{, x_{2}}$ we find that

$$
q_{, x_{1}}=q q_{, x_{2}}
$$

This is a very simple equation and its general solution can be found. We shall not follow this direction to determine $f\left(x_{1}, x_{2}\right)$ rather than change the form of equation $p\left(u, u_{x}\right)=0$ at $x=x_{0}$. This equation (in principle) implies either (a) $u_{x}=h(u)$, which implies $f=u_{x}-h(u)$ at $x=x_{0}$, or (b) $u=g\left(u_{x}\right)$, which implies $f=u-g\left(u_{x}\right)$ at $x=x_{0}$. It is now very easy to show that with the cases (a) and (b), when the corresponding $f$ 's are inserted in (27), we, respectively, obtain (a) $h^{\prime \prime}+2=0$, which implies $u_{x}+u^{2}+c_{1} u+c_{2}=0$ at $x=x_{0}$; and (b) $g^{\prime \prime}+2\left(g^{\prime}\right)^{3}=0$, which implies $u=$ constant (for $g^{\prime}=0$ ) and a special case of (a) (for $g^{\prime} \neq 0$ ). Hence we found all possible boundary conditions.

Remark 3.1: On the invariant surface $p(u, w)=0$ the system (24) turns into the Burgers'-like equation $u_{\tau}=u_{t t}-2\left(c_{1} u+c_{2}\right) u_{t}$, which is also integrable. ${ }^{9}$

\section{APPLICATIONS TO OTHER PARTIAL DIFFERENTIAL EQUATIONS}

In this section we shall apply our method to obtain compatible boundary conditions of some nonlinear partial differential equations. Let us start with the following system of equations:

$$
u_{t}=u_{2}+2 u^{2} v, \quad-v_{t}=v_{2}+2 u v^{2} .
$$

Letting $v \rightarrow u^{*}$ and $t \rightarrow i t$, the above system becomes the well-known nonlinear Schrödinger equation, where $*$ is the complex conjugation. It has the following recursion operator:

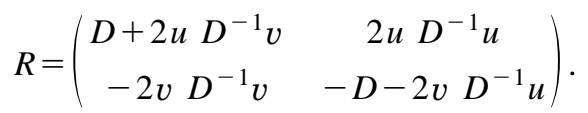

For the nonlinear Schrödinger equation, $\mathbf{R}$ takes the form

$$
\mathbf{R}=\left(\begin{array}{cccc}
-2 u \partial_{t}^{-1} v_{1} & 1+2 u \partial_{t}^{-1} v & 2 u \partial_{t}^{-1} u_{1} & -2 u \partial_{t}^{-1} u \\
\xi-2 u_{1} \partial_{t}^{-1} v_{1} & 2 u_{1} \partial_{t}^{-1} v & 2 u_{1} \partial_{t}^{-1} u_{1} & -2 u_{1} \partial_{t}^{-1} u \\
2 v \partial_{t}^{-1} v_{1} & -2 v \partial_{t}^{-1} v & -2 v \partial_{t}^{-1} u_{1} & -1+2 v \partial_{t}^{-1} u \\
2 v_{1} \partial_{t}^{-1} v_{1} & -2 v_{1} \partial_{t}^{-1} v & \eta-2 v_{1} \partial_{t}^{-1} u_{1} & 2 v_{1} \partial_{t}^{-1} u
\end{array}\right),
$$

where $\xi=\partial_{t}-2 u v, \eta=\partial_{t}+2 u v$, and $n_{0}=2$. Suppose that it admits a boundary condition of the following form:

$$
\left.u_{x}\right|_{x=0}=p^{1}(u, v),\left.\quad v_{x}\right|_{x=0}=p^{2}(u, v),
$$

compatible with the fourth-order symmetry. It means that the constraint (31) defines an invariant surface for this symmetry, presented as a system of four equations with four independent variables,

$$
\begin{gathered}
u_{\tau}=u_{t t}-2 u^{2} v_{t}-4 u v_{1} u_{1}+2 v u_{1}^{2}-2 u^{3} v^{2}, \\
u_{1, \tau}=u_{1, t t}-2 u^{2} v_{1 t}-2 u_{1}^{2} v_{1}-6 u^{2} v^{2} u_{1}-4 u v_{1} u_{t}+4 v u_{1} u_{t}+4 v u^{3} v_{1}, \\
v_{\tau}=-v_{t t}-2 v^{2} u_{t}+4 v u_{1} v_{1}-2 u v_{1}^{2}+2 v^{3} u^{2}, \\
v_{1, \tau}=-v_{1, t t}-2 v^{2} u_{1, t}+2 v_{1}^{2} u_{1}+6 v_{1} v^{2} u^{2}-4 v u_{1} v_{t}+4 u v_{1} v_{t}-4 v^{3} u u_{1} .
\end{gathered}
$$

One can check that the system (32) is compatible with the constraint $u_{1}=p^{1}(u, v), v_{1}=p^{2}(u, v)$ only if $p^{1}=c u$ and $p^{2}=c v$. Since the system (32) is of the form 


$$
\left(u, u_{1}, v, v_{1}\right)_{\tau}^{T}=\mathbf{R}^{2}\left(u, u_{1}, v, v_{1}\right)_{t}^{T},
$$

it follows from Proposition 2.2 that the constraints $u_{1}=c u, v_{1}=c v$ are compatible with every symmetry of even order. So the boundary conditions $\left.u_{x}\right|_{x=0}=c u,\left.v_{x}\right|_{x=0}=c v$ are compatible with such symmetries. Analytical properties of this boundary value problem are studied previously (see Refs. 4, 10, and 11) by means of the inverse scattering method.

Remark 4.1: On the invariant surface $u_{1}=c u, v_{1}=c v$ the system (32) is reduced to a system of two equations:

$$
\begin{gathered}
u_{\tau}=u_{t t}-2 u^{2} v_{t}-2 c^{2} u^{2} v-2 u^{3} v^{2}, \\
v_{\tau}=-v_{t t}-2 v^{2} u_{t}+2 c^{2} v^{2} u+2 v^{3} u^{2} .
\end{gathered}
$$

The integrability of these equations is shown in Ref. 5 (see p. 175). Under a suitable change of variables in it this system of two equations becomes the famous derivative nonlinear Schrödinger equation.

Among the nonlinear integrable equations, the Harry-Dym equation,

$$
u_{t}+u^{3} u_{3}=0
$$

is of special interest because its analytical properties are not typical. Using the symmetry approach we find a boundary condition of the form

$$
p\left(u, u_{1}, u_{2}\right)=0,
$$

compatible with the Harry-Dym equation. One has to notice that the transformation from the standard set of variables $u, u_{1}, u_{2}, u_{3}, \ldots$, to $u, u_{1}, u_{2}, u_{t}, u_{1, t}, u_{2, t}, \ldots$, is not regular. For instance, $u_{3}=-u_{t} / u^{3}$. It has a singular surface given by the equation $u=0$. So one should examine this surface separately. Since the Harry-Dym equation (34) as well as its higher-order symmetries possess the reflection symmetry $x \rightarrow-x, u \rightarrow-u, t \rightarrow t$ the trivial boundary condition $u(t, 0)=0$ is consistent with the integrability.

Suppose that the boundary value problem (34) and (35) is compatible with the ninth-order symmetry $u_{\tau}=u^{9} u_{g}+\cdots$. It means that the constraint $p(u, v, w)$ is consistent with following system of equations, equivalent to the ninth symmetry:

$$
u_{\tau}=f_{1}, \quad v_{\tau}=f_{2}, \quad w_{\tau}=f_{3},
$$

where $v=u_{x}, w=u_{x x}$, and $\left(f_{1}, f_{2}, f_{3}\right)^{T}=\mathbf{R}^{3}\left(u_{t}, v_{t}, w_{t}\right)^{T}$, where

$$
\mathbf{R}=\left(\begin{array}{ccc}
u w+u_{t} \partial_{t}^{-1} w & -u v-u_{t} \partial_{t}^{-1} v & u^{2}+u_{t} \partial_{t}^{-1} u \\
(1 / u) \partial_{t}+v w-u_{t} / u^{2}+v_{t} \partial_{t}^{-1} w & -v^{2}-v_{t} \partial_{t}^{-1} v & u v+v_{t} \partial_{t}^{-1} u \\
w^{2}+w_{t} \partial_{t}^{-1} w & (1 / u) \partial_{t}-v w-u_{t} / u^{2}-w_{t} \partial_{t}^{-1} v & u w+w_{t} \partial_{t}^{-1} u
\end{array}\right)
$$

The explicit expressions for $f_{2}, f_{3}$ are very long. Hence we give the explicit form only for the function $f_{1}$ :

$$
\begin{aligned}
f_{1}= & -u_{t t t}+3 u_{t t} u_{t} \frac{1}{u}-\frac{3}{2} u_{t t} u_{1} h-\frac{3}{2} \frac{u_{t}^{3}}{u^{2}}+\frac{3}{2} u u_{1, t t} h \\
& +\frac{3}{2} u u_{1, t} h_{t}-\frac{15}{16} u h^{2} h_{t}-\frac{5}{16} h^{3} u_{t}-\frac{3}{2} u_{1} u_{t} h_{t},
\end{aligned}
$$


where $h=2 u_{2} u-u_{1}^{2}$. Here one has two choices for the rank of Eq. (35). It is either one or two. The first choice does not lead to any regular invariant surface. The second gives

$$
\left.u_{x}\right|_{x=0}=c u,\left.\quad u_{x x}\right|_{x=0}=c^{2} u / 2 .
$$

Since the symmetry under consideration is of the form $u_{\tau}=R^{3} u_{t}$, where $R=u^{3} D^{3} u D^{-1}\left(1 / u^{2}\right)$ (see Ref. 12) is the recursion operator for the Harry-Dym equation, assuming $u \rightarrow$ const $\neq 0$ as $|x| \rightarrow \infty,{ }^{13}$ and taking this constant to be -1 without loss of generality, we can write the following corollary to Proposition 2.2.

Corollary: The boundary value problem (34) and (35) is compatible with every symmetry of the form $u_{\tau}=L\left(R^{3}\right) u_{t}$, where $L$ is a scalar polynomial with constant coefficients.

Remark 4.2: On the invariant surface $v=c u, w=c^{2} u / 2$ Eq. (37) takes the form

$$
u_{\tau}=-u_{t t t}+3 u_{t} u_{t t} / u-3 u_{t}^{3} u^{2} / 2
$$

equivalent to the $\mathrm{mKdV}$ equation.

The Korteweg de Vries equation $u_{t}=u_{3}+6 u_{1} u$ admits a recursion operator $R=D^{2}+4 u+2 u_{1} D^{-1}$, which may be represented in the form

$$
\mathbf{R}=\left(\begin{array}{ccc}
4 u+12 v \partial_{t}^{-1} u & 0 & 1+2 v \partial_{t}^{-1} \\
\partial_{t}+12 w \partial_{t}^{-1} u & -2 u & 2 w \partial_{t}^{-1} \\
2 w+12\left(u_{t}-6 u v\right) \partial_{t}^{-1} u & \partial_{t}-2 v & -2 u+2\left(u_{t}-6 u v\right) \partial_{t}^{-1}
\end{array}\right)
$$

It is not difficult to show that the system of equations $(u, v, w)_{\tau}=\mathbf{R}^{3}(u, v, w)_{t}$ admits an invariant surface $u=0, w=0$ on which the equation turns into the $\mathrm{mKdV}$ equation. It means that the boundary condition $u(t, x=0)=0, u_{x x}(t, x=0)=0$ is compatible with all symmetries of the form $\left(u_{\tau}, v_{\tau}, w_{\tau}\right)^{T}=H\left(\mathbf{R}^{3}\right)\left(u_{t}, v_{t}, w_{t}\right)^{T}$. Similarly, the mKdV equation $u_{t}=u_{3}+6 u^{2} u_{x}$ is compatible with the boundary condition $u(t, x=0)=0, u_{x}(t, x=0)=0$.

\section{APPLICATIONS TO DISCRETE CHAINS}

Consider an integrable nonlinear chain of the form

$$
u_{t}(n)=f(u(n-1), u(n), u(n+1)),
$$

with unknown function $u=u(n, t)$ depending on integer $n$ and real $t$. The natural set of dynamical variables serving the hierarchy of higher symmetries for the chain is the set $u(0), u( \pm 1)$, $u( \pm 2), \ldots$. However, it is more convenient for our aim to use the following unusual one, consisting of the variables $u(0), u(1)$ and all their $t$-derivatives. Transformations of these sets to each other are given by Eq. (41) itself and its differential consequences. In terms of new basic variables, every higher-order symmetry of this chain,

$$
u_{\tau}(n)=g(u(n-m), u(n-m-1), \ldots, u(n+m)),
$$

could be presented as a system of two partial differential equations,

$$
u_{\tau}=G_{1}\left(v, w, v_{1}, w_{1}, \ldots, v_{s}, w_{s}\right), \quad w_{\tau}=G_{2}\left(v, w, v_{1}, w_{1}, \ldots, v_{s}, w_{s}\right),
$$

where $v=u(0, t, \tau), w=u(1, t, \tau), v_{i}=\partial^{i} v / \partial t^{i}, w_{i}=\partial^{i} w / \partial x^{i}$.

Prescribe some boundary condition of the form

$$
u(0)=p(u(1), u(2), \ldots, u(k))
$$


to Eq. (40) to hold for all moments $t$. We shall call the boundary value problem (40), (43) consistent with the symmetry (41) if the constraint (43) defines an invariant surface for the system (42). Note that interconnection between the hierarchies of the commuting discrete chains and integrable partial differential equations is well known (see the survey ${ }^{5}$ ). An illustrative example of this kind of connections is related to the famous Volterra chain,

$$
u_{t}(n)=u(n)(u(n+1)-u(n-1)) .
$$

Its next symmetry is

$$
u_{\tau}(n)=u(n) u(n+1)[u(n)+u(n+1)+u(n+2)]-u(n) u(n-1)[u(n)+u(n-1)+u(n-2)],
$$

which might be represented as (Ref. 5, p. 123)

$$
v_{\tau}+v_{t t}=\left(2 v w+v^{2}\right)_{t}, \quad w_{\tau}-w_{t t}=\left(2 v w+w^{2}\right)_{t},
$$

under the substitution $u(0)=v, u(1)=w, u(-1)=w-v_{t} / v, u(2)=v+w_{t} / w, u(-2)=v$ $-\partial \ln u(-1) / \partial t$. Moreover, the full hierarchy of the Volterra chain is completely described by the hierarchy of the last system. According to the definition above the boundary value problem (43), (44) will be consistent with a symmetry of the Volterra chain if the constraint (43) describes an invariant surface for the same symmetry, represented as a system of partial differential equations. Let us examine invariant surfaces of the following system of partial differential equations:

$$
v_{\tau}=v_{t t t}+\left(3 v H^{2}-3 v_{t} H-2 v^{3}\right)_{t}, \quad w_{\tau}=w_{t t t}+\left(3 w H^{2}+3 w_{t} H-2 w^{2}\right)_{t},
$$

where $H=v+w$, which is exactly the higher-order symmetry for the Volterra chain (44) of the form

$$
\begin{aligned}
u_{\tau}(n)= & u(n) u(n+1)[u(n+2) u(n+3)+u(n) u(n+2)+u(n) u(n-1) \\
& \left.+u^{2}(n)+2 u(n+1) u(n+2)+u^{2}(n+2)+2 u(n) u(n+1)+u^{2}(n+1)\right] \\
& -u(n) u(n-1)\left[u(n) u(n+1)+u(n) u(n-2)+u(n-2) u(n-3)+u^{2}(n-2)\right. \\
& \left.+2 u(n) u(n-1)+u^{2}(n)+2 u(n-1) u(n-2)+u^{2}(n-1)\right] .
\end{aligned}
$$

It is easy to check that the only invariant surface of the form $v=$ const admissible by the system (43) is $v=0$. The corresponding boundary condition $u(0)=0$ is well studied (see Refs. 14 and 15).

Remark 5.1: On the invariant surface $v=0$ the system (46) reduces to the scalar equation

$$
w_{\tau}=w_{t t t}+3 w_{t t} w+3 w_{t}^{2}+3 w_{t} w^{2},
$$

which is nothing else but the next symmetry of the Burgers' equation. Moreover, the constraint is compatible with every generalized polynomial symmetry. On the invariant surface they are all reduced to the symmetries of the Burgers' equation. It is evident, for instance, that the system (46) turns into the Burgers' equation itself.

Suppose now that $v=p(w)$. Then one obtains that $p(w)=-w$. It gives rise to a boundary condition $u(0)=-u(1)$ compatible with the Volterra chain (see Ref. 16).

Remark 5.2: Under the constraint $v=-w$ the system (46) turns into the modified KdV equation,

$$
v_{\tau}=v_{t t t}+6 v^{2} v_{t} .
$$

It is not difficult to show that there is no any invariant surface of the form $v=p\left(w, w_{t}\right)$ such that $\partial p / \partial w_{t} \neq 0$ admissible with the system (46). 
For the case $v_{t}=p\left(v, w, w_{t}\right)$ calculations become very long so that here we utilized Mathematica 2.1 (we thank George Alekseev for his help with this calculations). Here $p$ has a form $p=(v / w) w_{t}+2 v(v+w)$, which produces the boundary condition $u(-1)=-u(0)-u(1)-u(2)$. The slight difference with (43) is overcome by the simple shift of the discrete variable $n$.

Using Proposition 2.1, it is easy to check that the invariant surface $v_{t}=(v / w) w_{t}+2 v(v+w)$ is compatible with every odd-order polynomial generalized symmetry of the system (46). It means that the boundary condition $u(-1)=-u(0)-u(1)-u(2)$ is compatible with the corresponding symmetries of the Volterra chain.

The well-known boundary condition $u^{2}(0)=1$ for the modified Volterra chain,

$$
u_{t}(n)=\left(1-u^{2}(n)\right)(u(n+1)-u(n-1)),
$$

defines the invariant surface $v^{2}=1$ for the following systems of equations:

$$
v_{\tau}+v_{t t}=2\left(\left(1-v^{2}\right) w\right)_{t}, \quad w_{\tau}-w_{t t}=2\left(\left(1-w^{2}\right) v\right)_{t}
$$

and

$$
\begin{gathered}
v_{\tau}+v_{t t t}=2\left(v\left(1-v^{2}\right)\left(3 w^{2}-1\right)-3 v w v_{t}\right)_{t}, \\
w_{\tau}+w_{t t t}=2\left(w\left(1-w^{2}\right)\left(3 v^{2}-1\right)+3 v w w_{t}\right)_{t},
\end{gathered}
$$

which are equivalent to the next symmetries of this chain:

$$
u_{\tau}(n)=\left(1-u^{2}(n)\right)\left(D_{-}-D_{+}\right)\left(1-u^{2}(n)\right)\left(D_{-}-D_{+}\right) u(n)
$$

and

$$
\begin{aligned}
u_{\tau}(n)= & \left(1-u^{2}(n)\right)\left(D_{-}-D_{+}\right)\left(1-u^{2}(n)\right)\left[\left(-D_{+}^{2}-D_{-}^{2}\right) u(n)+\left(D_{+}+D_{-}\right)\left(u^{2}(n) u(n+1)\right.\right. \\
& \left.\left.+u^{2}(n) u(n-1)+2 u(n-1) u(n) u(n+1)\right)\right] .
\end{aligned}
$$

Here $D_{+}, D_{-}$are the shift operators: $D_{+} u(n)=u(n+1), D_{-} u(n)=u(n-1) ; v=u(0), w=u(1)$ and other variables $u(n)$ are expressed through $v, w$, and their $t$-derivatives by means the chain and its differential consequences.

Remark 5.3: On the invariant surface $v^{2}=1$ the systems (47), (48) are reduced to the Burgers' equation and its third-order symmetry.

\section{CONDITION OF WEAK COMPATIBILITY}

It is easy to notice that any symmetry of Eq. (1) rewritten in terms of the nonstandard set of the dynamical variables turns into the equation containing $m-1$ extra variables $u_{1}, u_{2}, \ldots, u_{m-1}$. For instance, the fourth-order symmetry of the Burgers' equation,

$$
u_{\tau}=u_{4}+4 u_{3} u+10 u_{2} u_{1}+6 u_{2} u^{2}+12 u_{1}^{2} u+4 u_{1} u^{3},
$$

takes the following form:

$$
u_{\tau}=u_{t t}+2\left(w+u^{2}\right) u_{t}
$$

where $w=u_{1}$. To extend it to the closed form, it is enough to add one more equation obtained from the above equation by the differentiation with respect to $x$ and replacing $u_{2}=u_{t}-2 u w$. This is the general rule for integrable equations: One has to add $m-1$ more equations (to have a closed system of equations), expressing variables $u_{i \tau}, 1 \leqslant i \leqslant m-1$ through dynamical ones. But on the other hand, one may consider the single symmetry equation alone and suppose the extra variables 
are expressed interms of $u$ and its lower derivatives. Let us pose the question, for which choice of such expressions does the symmetry under consideration turn into an integrable equation? As an example let us consider the Burgers' equation. How should we choose the dependence $w=w(u)$, such that the equation $u_{\tau}=u_{t t}+2\left(w+u^{2}\right) u_{t}$ would be integrable? The only choice is $w=-u^{2}+c_{1} u+c_{2}$ (see Ref. 17). We will call the boundary conditions $u_{i}=u_{i}(u), x=0$ (obtained this way) for Eq. (1) as weakly compatible with the symmetry if these constraints are chosen to satisfy the requirement above; i.e., the equation for the $n$th symmetry written down in terms of the introduced variables turns into some integrable equation after replacing $u_{i}=u_{i}(u)$, $u_{i t}=u_{t}\left(\partial u_{i} / \partial u\right), \ldots$. So in the above case of the Burgers' equation only the condition $w(u)=-u^{2}+c_{1} u+c_{2}$ is weakly compatible with the fourth-order symmetry. As the remarks given above indicate, the compatibility of the condition with a symmetry implies the weak compatibility with it, but not vice versa. However, we conjecture that if the boundary condition is weakly compatible with at least three higher symmetries then the corresponding initial boundary value problem will be solvable by a suitable generalization of the inverse scattering method.

The following example for the Harry-Dym equation (34) seems to be intriguing. Let us represent the fifth-order symmetry,

$$
u_{\tau_{5}}=-\frac{1}{2} u^{3}\left(2 u_{5} u^{2}+10 u_{4} u_{1} u+10 u_{3} u_{2} u+5 u_{3} u_{1}^{2}\right)
$$

in the form $u_{\tau_{5}}=\frac{1}{2}(h u)_{t}$, where $h=2 u_{2} u-u_{1}^{2}$. Represent also the next two symmetries in the similar form:

$$
u_{\tau_{7}}=u_{t t} u_{1}-\frac{3}{2} u_{t} u_{1} u h+\frac{3}{8} u_{t}\left[3\left(h+u_{1}^{2}\right)^{2}-4 u_{1}^{2}\left(h+u_{1}^{2}\right)+u_{1}^{4}\right]-u u_{1 t t}+\frac{3}{8} u_{2 t} u h
$$

and $u_{\tau_{9}}=f_{1}$ [see Eq. (37)]. It is evident that for arbitrary function $F=F(u)$ the constraint $h=0$, $u_{1}=F(u)$ is weakly consistent with fifth and ninth symmetries, because the former takes the trivial form $u_{\tau_{5}}=0$ and the latter turns into the integrable equation (39). The seventh-order symmetry becomes $u_{\tau_{7}}=\left(S u_{t}\right)_{t}$, where $S=F-u F^{\prime}$. Thus, if for instance, $S=a=$ const or $S=1 /(\gamma u+\beta)^{2}$, one will have the equation $u_{\tau_{7}}=\left(S u_{t}\right)_{t}$, to be integrable (see Ref. 9, p. 129). Supposing $S(u)=a$ one can easily find that $u_{1}=c u+a, u_{2}=c^{2} u / 2+a c+a^{2} / 2 u$. It leads to the following boundary condition $u_{x}=c u+a, u_{x x}=u_{x}^{2} / 2 u$, at $x=0$ for the Harry-Dym equation, which coincides with (38) if $a=0$. In the case $S=1 /(\gamma u+\beta)^{2}$ to find $F$, one has to integrate the ordinary differential equation $F(u)-u F^{\prime}(u)=S$.

\section{ACKNOWLEDGMENTS}

This work has been supported by the Turkish Scientific and Technical Research Council (TUBITAK). M.G. is an associate member of Turkish Academy of Sciences (TUBA). I.H. thanks TUBITAK and the Russian Foundation for Fundamental Research (Grant No. 93-011-165) for their partial support, and Bilkent University for warm hospitality.

\footnotetext{
${ }^{1}$ B. Gürel, M. Gürses, and I. Habibullin, Phys. Lett. A 190, 231 (1994).

${ }^{2}$ I. T. Habibullin, Phys. Lett. A 178, 369 (1993).

${ }^{3}$ A. S. Fokas and A. R. Its, Phys. Rev. Lett. 68, 3117 (1992).

${ }^{4}$ E. K. Sklyanin, Funct. Anal. Prilozh. 21, 86 (1987).

${ }^{5}$ A. V. Mikhailov, V. V. Sokolov, and A. B. Shabat, in What is Integrability? (Springer, Berlin, 1991), p. 115.

${ }^{6}$ M. Gürses, A. Karasu, and A. Satir, in Nonlinear Evolution Equations and Dynamical Systems, NEEDS'91, edited by M. Boiti, L. Martinelli, and F. Pempinelli (World Scientific, Singapore, 1992).

${ }^{7}$ M. Gürses and A. Karasu, J. Math. Phys. (to appear).

${ }^{8}$ P. J. Olver, Applications of Lie Groups to Differential Equations (Springer, Berlin, 1986).

${ }^{9}$ A. S. Fokas, J. Math. Phys. 21, 1318 (1980); SIAM 77, 253 (1987).

${ }^{10}$ P. N. Bibikov and V. O. Tarasov, Teor. Mat. Fiz. 79, 334 (1989).

${ }^{11}$ I. T. Khabibullin, Teor. Mat. Fiz. 86, 130 (1991).

${ }^{12}$ M. Leo, R. A. Leo, G. Soliani, and L. Solombrino, Lett. Nuovo Cimento 38, 45 (1983).
} 
${ }^{13}$ M. Leo, R. A. Leo, G. Soliani, L. Solombrino, and L. Martina, Phys. Rev. D 27, 1406 (1983).

${ }^{14}$ M. Kac and P. van Moerbeke, Proc. Natl. Acad. Sci. USA 72, 2879 (1975).

${ }^{15}$ A. K. Common and S. T. Hafez, J. Phys. A 23, 455 (1990).

${ }^{16}$ A. B. Shabat and R. I. Yamilov, Leningrad Math. J. 2, 2 (1991).

${ }^{17}$ S. I. Svinolupov, Phys. Lett. A 135, 32 (1989). 